OPEN ACCESS

Edited by:

Oreste Gualillo,

Servicio Gallego de Salud, Spain

Reviewed by:

Carsten Culmsee,

Philipps University of Marburg,

Germany

Abdu Adem,

United Arab Emirates University,

United Arab Emirates

*Correspondence:

Sandra M. Cardoso cardoso.sandra.m@gmail.com

Specialty section:

This article was submitted to Integrative Physiology, a section of the journal

Frontiers in Physiology

Received: 24 January 2018 Accepted: 16 April 2018 Published: 09 May 2018

Citation:

Cardoso SM and Empadinhas N (2018) The Microbiome-Mitochondria

Dance in Prodromal Parkinson's Disease. Front. Physiol. 9:471. doi: 10.3389/fphys.2018.00471

\section{The Microbiome-Mitochondria Dance in Prodromal Parkinson's Disease}

\author{
Sandra M. Cardoso ${ }^{1,2 *}$ and Nuno Empadinhas ${ }^{1}$ \\ ${ }^{1}$ Center for Neuroscience and Cell Biology, University of Coimbra, Coimbra, Portugal, ${ }^{2}$ Institute of Cellular and Molecular \\ Biology, Faculty of Medicine, University of Coimbra, Coimbra, Portugal
}

The brain is an immunologically active organ where neurons and glia cells orchestrate complex innate immune responses against infections and injuries. Neuronal responses involve Toll-like or Nod-like receptors and the secretion of antimicrobial peptides and cytokines. The endosymbiotic theory for the evolutionary origin of mitochondria from primitive bacteria, suggests that they may have also retained the capacity to activate neuronal innate immunity. In fact, it was shown that mitochondrial damage-associated molecular patterns could signal and activate innate immunity and inflammation. Moreover, the mitochondrial cascade hypothesis for sporadic Parkinson's disease (PD) argues that altered mitochondrial metabolism and function can drive neurodegeneration. Additionally, a neuroinflammatory signature with increased levels of pro-inflammatory mediators in PD affected brain areas was recently detected. Herein, we propose that a cascade of events initiating in a dysbiotic gut microbiome drive the production of toxins or antibiotics that target and damage mitochondria. This in turn activates neuronal innate immunity and triggers sterile inflammation phenomena that culminate in the neurodegenerative processes observed in the enteric and in the central nervous systems and that ultimately lead to Parkinson's disease.

Keywords: mitochondria, bacteria, microbiome, neuronal innate immunity, Parkinson's disease

\section{PARKINSON'S DISEASE OVERVIEW}

Parkinson's disease (PD), the most frequent neurodegenerative movement disorder, is characterized by severe loss of midbrain dopaminergic neurons in the $\mathrm{SNpc}$ and by the presence of intra-cytoplasmic inclusions of aggregated SNCA, known as LBs (Obeso et al., 2010). Sporadic $\mathrm{PD}(\mathrm{sPD})$ is a multifactorial disorder that evolves over decades without any motor complications. PD has a long prodromal phase during which several other symptoms develop, namely related to olfactory impairment, sleep disturbances, and depression (Reichmann et al., 2009). Another common underlying symptom described for the prodromal phase in PD patients is GI dysfunction

\footnotetext{
Abbreviations: AMP, antimicrobial peptides; CNS, central nervous system; DAMP, damage-associated molecular pattern; ENS, enteric nervous system; FPR, formyl peptide receptors; GI, gastrointestinal; IL, interleukin; LBs, Lewy bodies; LPS, lipopolysaccharide; mtDNA, mitochondrial DNA; mtROS, mitochondrial produced reactive oxygen species; NLR, NODlike receptors; Nlrp3, NLR family pyrin domain containing 3; NOD, nucleotide-binding oligomerization domain-like; PAMP, pathogen-associated molecular pattern; PD, Parkinson's disease; PET, positron emission tomography; PRR, pattern recognition receptor; SNCA, alpha-synuclein; SNpc, substantia nigra pars compact; TIR, Toll/interleukin-1 receptor; TLRs, Toll-like receptors; TNF $\alpha$, tumor necrosis factor $\alpha$.
} 
that also includes dysphagia, gastroparesis, and severe constipation (Pfeiffer, 2003). These symptoms correlate with Braak staging whereas SNCA-immunopositive Lewy neurites and LBs target specific induction sites: initially in the dorsal motor nucleus of the glossopharyngeal and vagal nerves and in the anterior olfactory nucleus (Braak et al., 2003a).

Because LBs are also detected in the ENS of earliest and asymptomatic stage patients (Natale et al., 2011), the GI tract was proposed as an early target of PD pathology. Despite this, several evidences resulting from brain autopsy, animal models and cellular studies show that PD neurodegeneration involves multiple cellular processes, including mitochondrial dysfunction, oxidative stress, proteasomal and autophagic impairments and neuroinflammation (Olanow, 2007).

\section{MITOCHONDRIAL INVOLVEMENT IN PARKINSON'S DISEASE ETIOLOGY}

Mitochondria host biochemical reactions essential for normal cell functioning, namely energy production and maintenance of redox homeostasis (Lobet et al., 2015). Mitochondria were associated to sPD pathology when deficits in mitochondrial $\mathrm{NADH}$ dehydrogenase (complex I) activity were identified in the $\mathrm{SNpc}$ of post-mortem PD patient's brains and in their platelets (Cardoso, 2011). Our group demonstrated that dysfunctional mitochondria from PD patients' trigger several pathogenic features observed in PD subject brains, such as the generation of protein aggregates (LBs "like") (Esteves et al., 2009), microtubule disassembly, disruption of intracellular trafficking (Esteves et al., 2010) and accumulation of autophagosomes and autophagic substrates (Arduíno et al., 2012).

The complex I inhibitors 1-methyl-4-phenyl-1,2,3,6tetrahydropyridine and rotenone are widely used as in vitro and in vivo models of $\mathrm{PD}$ given that they recapitulate the main features of the disease (Meredith and Rademacher, 2011; Xiong et al., 2012). Mitochondrial dysfunction in PD tissues and models is also characterized by a decrease in mitochondrial membrane potential (Mann et al., 1992; Esteves et al., 2008), and by an increase in mitochondrial pool fragmentation and cristae disruption (Baloyannis et al., 2006; Santos et al., 2015). Accordingly, at a functional level, brain bioenergetics is compromised in PD where PET scans show decreased glucose utilization in PD individuals in the occipital cortex compared to control individuals (Schapira, 2008).

\section{RELEVANCE OF A BACTERIAL ORIGIN OF MITOCHONDRIA}

After exposure to a new pathogen, our innate immune system protects us from infection. Innate immune responses are not specific to a particular pathogen and depend on the recognition of several conserved features of pathogens (Ward and Rosenthal, 2014). The innate immune response relies on PRRs to identify PAMPs, many of which are normal components of bacterial cells (Pallen, 2011).
Mitochondria share a common ancestor with Alphaproteobacteria and so proposed to be derived from ancestral bacterial endosymbiosis. The evidence supports a common origin for mitochondria and bacteria related to the Rickettsiales that have extremely reduced genomes and have obligate intracellular lifestyles (Fitzpatrick et al., 2006). mtDNA shares features with the genome of Rickettsia prowazekii but the similarities between bacteria and mitochondria extend beyond the abundance in the distinctive lipid cardiolipin in the inner membrane, to the numerous small molecule transport systems and to an electron transport chain that pumps protons across the inner mitochondrial membrane with the resulting proton motive force driving ATP synthesis via the $\mathrm{F}_{1}$ ATP synthase. Additionally, both the matrix of mitochondria and the cytosol of bacteria contain DNA, tRNA, ribosomes, and numerous soluble enzymes; both reproduce by binary fission and bear a $\mathrm{N}$-formylmethionine start residue in their proteins. Remarkably, some bacterial PAMPs persist in mitochondria, such as formyl peptides that activate FPRs and unmethylated CpG dinucleotides, which activate TLR. Therefore the innate immune system does indeed recognize mitochondrial bacterial motifs, also called DAMPs. Upon mitochondrial release of DAMPs a sterile inflammation is activated that mimics the response to infection (Pallen, 2011).

\section{MITOCHONDRIA DAMPS TRIGGERS INNATE IMMUNITY}

The innate immune response can also be triggered by tissue damage independently of infection, a process also referred to as sterile inflammation, during which damaged cells release endogenous messengers known as DAMPs that are able to activate TLRs (Wilkins et al., 2017). At least 13 mammalian TLR isoforms are known, and each is capable of recognizing certain types of PAMPs or DAMPs (Takeuchi and Akira, 2010). Since mitochondria are an important source of DAMPs, the release of these mitochondrial DAMPs upon injury activates the innate immune system (Taanman, 1999). mtDNA is similar to bacterial DNA containing CpG motifs, which activate the TLR9 (Taanman, 1999; Zhang et al., 2010). Moreover, mitochondrial protein synthesis is initiated with the residue $N$-formylmethionine, similar to bacterial protein synthesis (Rabiet et al., 2007). The resulting bacterial $N$-formylated peptides are known to act as PAMPs by binding and activating G protein-coupled FPRs (Gurung et al., 2015), while the mitochondrial $N$-formylated peptides act as DAMPs through activation of the FPR1 (Taanman, 1999). Several studies have now described a crucial role for mitochondria in the regulation and activation of NLR specifically the Nlrp3 inflammasome (Latz et al., 2013). The inflammasomes are intracellular molecular platforms activated upon cellular infection or sterile stressors, which activate the pro-inflammatory cytokines interleukin-1 $\beta$ (IL-1 $\beta$ ) and IL-18, to trigger cell death (reviewed in Zhou et al., 2011). A variety of insults, resulting from cellular infection or stress, can promote mitochondrial dysfunction and activate the Nlrp3 inflammasome (Schroder and Tschopp, 2010; Latz 
et al., 2013). While initial studies showed that mitochondrial dysfunction and mtROS production are necessary for Nlrp3 inflammasome activation (Nakahira et al., 2011; Shimada et al., 2012), further evidence has shown that mtDNA translo cation to the cytosol plays an active role in this process, where it can directly bind to and activate the Nlrp3 inflammasome (de Andrade Rosa et al., 2006). In addition, the mitochondrial lipid cardiolipin is also essential for Nlrp3 inflammasome activation, by directly binding to Nlrp3, downstream of mitochondrial dysfunction (Iyer et al., 2013). Altogether, by sensing mitochondrial DAMPs, the Nlrp3 inflammasome plays a critical role in integrating mitochondrial dysfunction in a pro-inflammatory signaling response, thus explaining the association of mitochondrial damage with inflammatory diseases.

Despite the great number of studies describing mitochondria as a source of DAMPs, the potential for mitochondrial DAMPs to trigger, or exacerbate, inflammation in the brain is now being explored. In recent studies, this potential was tested by treating different brain cell types with mitochondrial components, and measuring markers of inflammation. Neuronal and microglial cell lines exposed to mitochondrial lysates displayed increased markers of inflammation, with mtDNA being identified as the candidate DAMP responsible for the inflammatory changes (Wilkins et al., 2015). Recently, it was observed that stereotactic injection of mitochondrial lysates or purified mtDNA into animal hippocampi induced pro-inflammatory modifications (Wilkins et al., 2016), such as increased levels of hippocampal TNF $\alpha$ mRNA, glial fibrillary acidic protein and NFKB phosphorylation in the cortex.

\section{NEURONAL INNATE IMMUNITY ACTIVATION IN PARKINSON'S DISEASE}

Innate immunity reacts to different insults that may challenge the integrity of the CNS. This process is initiated by receptors of the TLR family that are activated by PAMPs or DAMPs. In the brain, this response is considered to be mediated by microglial cells, the major antigen-presenting cells in the CNS. Nevertheless, neurons also express critical TIR domain-containing adaptors that transduce signals of TLR, namely TLR1, TLR2, TLR3, TLR4, TLR7, and TLR9, and regulate the expression of various cytokines (Liu et al., 2014). Indeed, TLR3 and 7, localized in the neuronal endosomal compartment, play a role in neurite outgrowth. In neurons, TLR9 mainly found in the ER (Shintani et al., 2014), reduces the calcium transfer to the mitochondria promoting autophagy and cell survival (de Bernard and Rizzuto, 2014; Shintani et al., 2014). Others also found that TLR4 signaling regulates axonal growth, neuronal plasticity and even adult neurogenesis (Okun et al., 2011). Moreover, in vitro activation of neuronal TLR4 by LPS induces a strong expression of neuronal chemokines. These data revealed that neuronal TLR4 activation may play a central role in the onset of innate immunity during CNS infection or harm (Leow-Dyke et al., 2012). It is assumed that the cytokines produced by neurons may be just enough to recruit and activate local microglia without causing global brain inflammation. So it is perceived that also neuronal cells are able to mount an innate immune response. In fact, CNS neurons can be crucial sensors of infection since they respond to LPS by producing pro-inflammatory chemokines that in turn lead to activation of endothelial cells (Leow-Dyke et al., 2012). Interestingly, also ENS neurons respond to LPS and produce TNF- $\alpha$ (Coquenlorge et al., 2014). Regardless of PD being characterized by a slow and progressive degeneration of dopaminergic neurons in the $\mathrm{SNpc}$, the cause of this neuronal loss is still poorly understood. Most relevant is the possibility that genetically determined age-dependent decline in mitochondrial function of the PD-typical pathologic cascade, gut bacteria or even their metabolites targeting the mitochondria, could activate innate immunity in dopaminergic neurons, due to the exposure of DAMPs, and in this way contribute to low-grade inflammation.

It was shown in PD cellular and animal models that mitochondrial network is highly fragmented. Mitochondrial fission is a prerequisite for the selective targeting of dysfunctional mitochondria for degradation by the lysosome in a process called mitophagy (Santos et al., 2015; Esteves et al., 2018). Nevertheless, it was recently proven that mitochondrial fission leads to the exposure of the inner membrane phospholipid, cardiolipin, which serves an important defensive function for the elimination of damaged mitochondria (Chu et al., 2013). Since cardiolipin is only found in mitochondrial and bacterial membranes it is considered a mitochondrial-derived DAMP that is detected by the Nlrp3 (He et al., 2016). NLR and TLR activation trigger the production of pro-inflammatory cytokines and AMPs (Lampron et al., 2013). Recently, it was also demonstrated that PD-associated SNCA proteins might be involved in the innate immunity response (Stolzenberg et al., 2017). It was proven that SNCA production mobilizes immune defenses against pathogens and the levels of mRNA of inflammatory cytokines in colonic biopsies from PD patients correlates with disease duration (Devos et al., 2013). Moreover, it was described that SNCA inserts in the mitochondrial membrane leading to mitochondrial dysfunction and also potentiate its fragmentation (Shen et al., 2014). These results seem to point to a positive feedback loop whereas mitochondrial dysfunction increases cardiolipin exposure, which in turn activates neuronal innate immunity. The question still remains on what is the role of SNCA under these conditions, one possibility being its involvement in the innate immunity pathway culminating in the potentiation of mitochondrial dysfunction.

\section{MITOCHONDRIA: TARGETS OF THE HUMAN MICROBIOME}

Mitochondria play a key in the regulation of many immune functions through metabolic control, calcium homeostasis and ROS production, thus assisting host defenses against pathogens (Lobet et al., 2015). However, many bacteria have evolved several different types of effectors and mechanisms that target the mitochondria precisely as a strategy to circumvent mitochondriadependent surveillance. In light of the endosymbiotic theory it 
can be argued that these mechanisms may have evolved in the ancestor bacteria as a strategy to gain competitive advantage in overpopulated environments. The mammalian gut harbors highly complex microbial communities with constantly balanced microbe-microbe and microbe-host interactions involving cooperative and competitive mechanisms to maximize the available resources and a shared co-existence (Sana et al., 2017). Some microbial species take advantage of nutrients produced by others in the community, while others target and kill their competitors by releasing toxic metabolites or by secreting effectors. We propose that gut dysbiosis increasingly associated to $\mathrm{PD}$ may, in the complex and competitive gut ecosystem, promote unrestrained and chronic production of microbial toxins that also target the mitochondria of ENS and CNS neurons (Figure 1). Indeed, PD biopsy studies confirmed the presence of LBs in organs innervated by the vagus nerve (Cersosimo et al., 2013), indicating this as the obvious route for disease progression between the gut and the brain. This led to the hypothesis that an exogenous toxin or pathogen can trigger the disease and spread via retrograde axonal vagus transport from the ENS to the CNS, and that the GI symptoms in the vast majority of $\mathrm{PD}$ patients are pre-motor manifestations of the disease (Braak et al., 2003a,b; Hawkes et al., 2007). Indeed, full truncal vagotomy significantly decreased the risk for subsequent $\mathrm{PD}$, which suggests that the vagus nerve is indeed a key player in PD pathogenesis, again corroborating the involvement of an enteric pathogen or toxin in disease progression (Svensson et al., 2015). Recently, the development of SNCA pathology in genetically susceptible mice was shown to require the presence of gut microbiota, as evidenced by the limited pathophysiology observed in germ-free and antibiotictreated susceptible mice, although the effect of antibiotics on the mitochondria was not evaluated. Remarkably, administration of certain microbial metabolites to genetically modified germ free mice reproduced major features of the disease, comparable to PD induced in mice with a complex microbiota (Sampson et al., 2016). Although PD gut microbiota signatures have recently begun to emerge, their functional interpretation still remains largely elusive (Hill-Burns et al., 2017; Scheperjans, 2018).

Many toxins produced by eventual gut microbes can damage mitochondria. Some Clostridium difficile strains secrete toxins that inhibit the mitochondrial ATP-sensitive potassium channels, drive mitochondrial membrane hyper-polarization, apoptosis and disruption of the gut epithelial barrier (Matarrese et al., 2007). Vibrio cholera secretes a toxin (VopE) that inhibits mitochondrial network reorganization (Suzuki et al., 2014). Although these toxins have not been identified in commensal

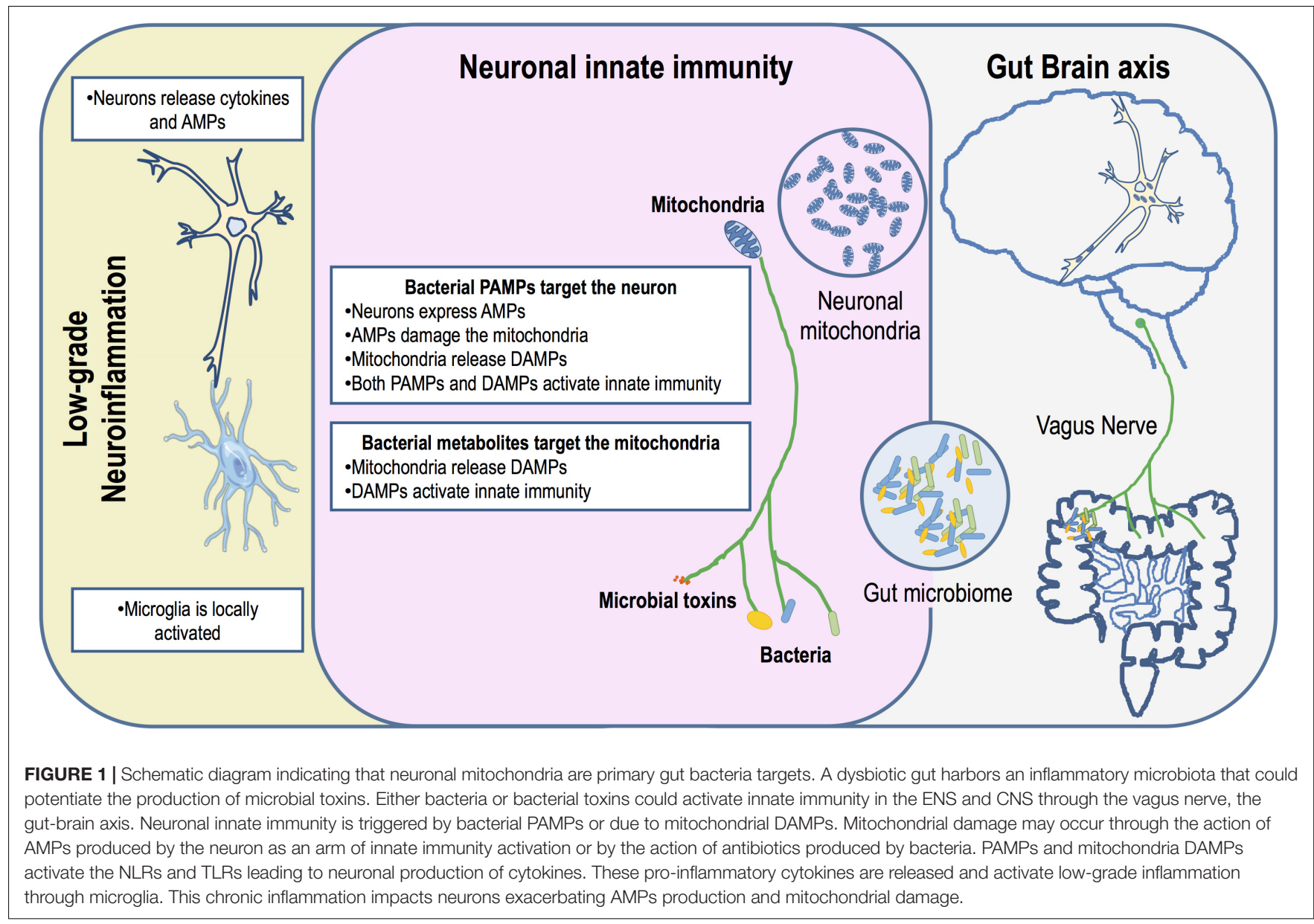


microbiota, gene clusters for their biosynthesis may be present and drive the synthesis of related toxic compounds that may impact mitochondria. Hypotheses for the etiology of PD pathology based on chronic exposure to environmental toxins have been proposed as the underlying cause for PD onset (Tanner et al., 2011). In theory, the gut microbiota might also represent the "environmental" source of toxins to which the host would be exposed. In addition to the above examples of toxins produced by pathogenic gut microbiota, low molecular weight antibiotics of different classes are also known to induce mitochondrial dysfunction and oxidative damage with pathological consequences (Kalghatgi et al., 2013). Some classes of antibiotics target the bacterial protein synthesis machinery and inadvertently also the mitochondrial ribosomes (mitoribosomes) with potentially severe side effects in the host (Hobbie et al., 2008; Qian and Guan, 2009). The mitochondrial protein synthesis apparatus is similar to that of bacteria as a result of a shared origin and later endosymbiosis. Consequently, mitochondrial ribosomes are frequently unintended off-targets of antibiotics such as the aminoglycosides directed to bacterial ribosomes (Hobbie et al., 2008). Actually, sensitivity to a given antibiotic is likely a multifactorial trait but the genetic makeup of sensitive individuals, including the observed higher mutation rates in mtDNA accumulated as a consequence of aging, may also be a major contributing factor (Qian and Guan, 2009; Pacheu-Grau et al., 2013). Considering that most of the known antibiotics in use since the 1940's are of microbial origin and more prominently produced by members of the phyla Actinobacteria and Firmicutes as well as by some Fungi, is it reasonable to anticipate that some

\section{REFERENCES}

Arduíno, D. M., Esteves, A. R., Cortes, L., Silva, D. F., Patel, B., Grazina, M., et al. (2012). Mitochondrial metabolism in Parkinson's disease impairs quality control autophagy by hampering microtubule-dependent traffic. Hum. Mol. Genet. 21, 4680-4702. doi: 10.1093/hmg/dds309

Baloyannis, S. J., Costa, V., and Baloyannis, I. S. (2006). Morphological alterations of the synapses in the locus coeruleus in Parkinson's disease. J. Neurol. Sci. 25, 35-41. doi: 10.1016/j.jns.2006.05.006

Braak, H., Del Tredici, K., Rüb, U., de Vos, R. A., Jansen Steur, E. N., and Braak, E. (2003a). Staging of brain pathology related to sporadic Parkinson's disease. Neurobiol. Aging 24, 197-211. doi: 10.1016/S0197-4580(02)00 065-9

Braak, H., Rüb, U., Gai, W. P., and Del Tredici, K. (2003b). Idiopathic Parkinson's disease: possible routes by which vulnerable neuronal types may be subject to neuroinvasion by an unknown pathogen. J. Neural Transm. 110, 517-536.

Cardoso, S. M. (2011). The mitochondrial cascade hypothesis for Parkinson's disease. Curr. Pharm. Des. 17, 3390-3397. doi: 10.2174/13816121179807 2508

Cersosimo, M. G., Raina, G. B., Pecci, C., Pellene, A., Calandra, C. R., Gutiérrez, C., et al. (2013). Gastrointestinal manifestations in Parkinson's disease: prevalence and occurrence before motor symptoms. J. Neurol. 260, 1332-1338. doi: 10.1007/s00415-012-6801-2

Chu, C. T., Ji, J., Dagda, R. K., Jiang, J. F., Tyurina, Y. Y., Kapralov, A. A., et al. (2013). Cardiolipin externalization to the outer mitochondrial membrane acts as an elimination signal for mitophagy in neuronal cells. Nat. Cell Biol. 15, 1197-1205. doi: 10.1038/ncb2837

Coquenlorge, S., Duchalais, E., Chevalier, J., Cossais, F., Rolli-Derkinderen, M., and Neunlist, M. (2014). Modulation of lipopolysaccharide-induced neuronal members of the gut microbiota may possess the genetic resources to synthesize other antibiotics or antimicrobials including toxins that may target their distantly related counterparts, the mitochondria. Indeed, unknown antimicrobials remain hidden in the largely unexplored human microbiome (Donia et al., 2014). Although their identity and effects in mitochondria with possible damage eventually leading to activation of innate immunity have not been addressed, the enormous biosynthetic potential for metabolites impacting microbes clearly indicates that we haven't seen but the tip of the mediators regulating the complex microbial interactions within us, and which might foster mitochondrial damage and neurodegenerative processes.

\section{AUTHOR CONTRIBUTIONS}

All authors listed have made a substantial, direct and intellectual contribution to the work, and approved it for publication.

\section{FUNDING}

Work in our laboratories was supported by Fundação para a Ciência e a Tecnologia (FCT) and by EU-FEDER funding through the Operational Competitiveness Program-COMPETE grant UID/NEU/04539/2013, by the European Regional Development Fund, Centro 2020 Regional Operational Program (CENTRO-01-0145-FEDER-000012-HealthyAging2020), and by Prémio Santa Casa Neurociências Mantero Belard MB-40-2016.

response by activation of the enteric nervous system. J. Neuroinflammation 11, 202. doi: 10.1186/s12974-014-0202-7

de Andrade Rosa, I., Einicker-Lamas, M., Roney Bernardo, R., Previatto, L. M., Mohana-Borges, R., Morgado-Diaz, J. A., et al. (2006). Cardiolipin in hydrogenosomes: evidence of symbiotic origin. Eukaryot. Cell 5, 784-787. doi: 10.1128/EC.5.4.784-787.2006

de Bernard, M., and Rizzuto, R. (2014). Toll-like receptors hit calcium. EMBO Rep. 15, 468-469. doi: 10.1002/embr.201438685

Devos, D., Lebouvier, T., Lardeux, B., Biraud, M., Rouaud, T., Pouclet, H., et al. (2013). Colonic inflammation in Parkinson's disease. Neurobiol. Dis. 50, 42-48. doi: 10.1016/j.nbd.2012.09.007

Donia, M. S., Cimermancic, P., Schulze, C. J., Wieland Brown, L. C., Martin, J., Mitreva, M., et al. (2014). A systematic analysis of biosynthetic gene clusters in the human microbiome reveals a common family of antibiotics. Cell 158, 1402-1414. doi: 10.1016/j.cell.2014.08.032

Esteves, A. R., Arduíno, D. M., Silva, D. F., Viana, S. D., Pereira, F. C., and Cardoso, S. M. (2018). Mitochondrial metabolism regulates microtubule acetylome and autophagy trough sirtuin-2: impact for parkinson's disease. Mol. Neurobiol. 55, 1440-1462. doi: 10.1007/s12035-017-0420-y

Esteves, A. R., Arduíno, D. M., Swerdlow, R. H., Oliveira, C. R., and Cardoso, S. M. (2009). Oxidative stress involvement in alpha-synuclein oligomerization in Parkinson's disease cybrids. Antioxid. Redox Signal. 11, 439-448. doi: 10.1089/ARS.2008.2247

Esteves, A. R., Arduíno, D. M., Swerdlow, R. H., Oliveira, C. R., and Cardoso, S. M. (2010). Microtubule assembly as a potential therapeutic target in Parkinson's disease. Front. Aging Neurosci. 4:5.

Esteves, A. R., Domingues, A. F., Ferreira, I. L., Januario, C., Swerdlow, R. H., Oliveira, C. R., et al. (2008). Mitochondrial function in Parkinson's disease cybrids containing an nt2 neuron-like nuclear background. Mitochondrion 8 , 219-228. doi: 10.1016/j.mito.2008.03.004 
Fitzpatrick, D. A., Creevey, C. J., and McInerney, J. O. (2006). Genome phylogenies indicate a meaningful alpha-proteobacterial phylogeny and support a grouping of the mitochondria with the Rickettsiales. Mol. Biol. Evol. 23, 74-85. doi: $10.1093 / \mathrm{molbev} / \mathrm{msj} 009$

Gurung, P., Lukens, J. R., and Kanneganti, T. D. (2015). Mitochondria: diversity in the regulation of the NLRP3 inflammasome. Trends Mol. Med. 21, 193-201. doi: 10.1016/j.molmed.2014.11.008

Hawkes, C. H., Del Tredici, K., and Braak, H. (2007). Parkinson's disease: a dualhit hypothesis. Neuropathol. Appl. Neurobiol. 33, 599-614. doi: 10.1111/j.13652990.2007.00874.x

He, Y., Hara, H., and Núñez, G. (2016). Mechanism and regulation of NLRP3 inflammasome activation. Trends Biochem. Sci. 41, 1012-1021. doi: 10.1016/j. tibs.2016.09.002

Hill-Burns, E. M., Debelius, J. W., Morton, J. T., Wissemann, W. T., Lewis, M. R., Wallen, Z. D., et al. (2017). Parkinson's disease and Parkinson's disease medications have distinct signatures of the gut microbiome. Mov. Disord. 32, 739-749. doi: 10.1002/mds.26942

Hobbie, S. N., Akshay, S., Kalapala, S. K., Bruell, C. M., Shcherbakov, D., and Böttger, E. C. (2008). Genetic analysis of interactions with eukaryotic rRNA identify the mitoribosome as target in aminoglycoside ototoxicity. Proc. Natl. Acad. Sci. U.S.A. 105, 20888-20893. doi: 10.1073/pnas.0811258106

Iyer, S. S., He, Q., Janczy, J. R., Elliott, E. I., Zhong, Z., Olivier, A. K., et al. (2013). Mitochondrial cardiolipin is required for Nlrp3 inflammasome activation. Immunity 39, 311-323. doi: 10.1016/j.immuni.2013.08.001

Kalghatgi, S., Spina, C. S., Costello, J. C., Liesa, M., Morones-Ramirez, J. R., Slomovic, S., et al. (2013). Bactericidal antibiotics induce mitochondrial dysfunction and oxidative damage in Mammalian cells. Sci. Transl. Med. 5:192ra85. doi: 10.1126/scitranslmed.3006055

Lampron, A., Elali, A., and Rivest, S. (2013). Innate immunity in the CNS: redefining the relationship between the CNS and its environment. Neuron 78 , 214-232. doi: 10.1016/j.neuron.2013.04.005

Latz, E., Xiao, T. S., and Stutz, A. (2013). Activation and regulation of the inflammasomes. Nat. Rev. Immunol. 13, 397-411. doi: 10.1038/nri3452

Leow-Dyke, S., Allen, C., Denes, A., Nilsson, O., Maysami, S., Bowie, A. G., et al. (2012). Neuronal toll-like receptor 4 signaling induces brain endothelial activation and neutrophil transmigration in vitro. J. Neuroinflammation 9:230. doi: 10.1186/1742-2094-9-230

Liu, H. Y., Chen, C. Y., and Hsueh, Y. P. (2014). Innate immune responses regulate morphogenesis and degeneration: roles of Toll-like receptors and Sarm1 in neurons. Neurosci. Bull. 30, 645-654. doi: 10.1007/s12264-014-1445-5

Lobet, E., Letesson, J. J., and Arnould, T. (2015). Mitochondria: a target for bacteria. Biochem. Pharmacol. 94, 173-185. doi: 10.1016/j.bcp.2015.02.007

Mann, V. M., Cooper, J. M., Krige, D., Daniel, S. E., Schapira, A. H., and Marsden, C. D. (1992). Brain, skeletal muscle and platelet homogenate mitochondrial function in Parkinson's disease. Brain 115, 333-342. doi: 10.1093/brain/115. 2.333

Matarrese, P., Falzano, L., Fabbri, A., Gambardella, L., Frank, C., Geny, B., et al. (2007). Clostridium difficile toxin B causes apoptosis in epithelial cells by thrilling mitochondria. Involvement of ATP-sensitive mitochondrial potassium channels. J. Biol. Chem. 282, 9029-9041. doi: 10.1074/jbc.M607614200

Meredith, G. E., and Rademacher, D. J. (2011). MPTP mouse models of Parkinson's disease: an update. J. Parkinsons Dis. 1, 19-33. doi: 10.3233/JPD-2011-11023

Nakahira, K., Haspel, J. A., Rathinam, V. A., Lee, S. J., Dolinay, T., Lam, H. C., et al. (2011). Autophagy proteins regulate innate immune responses by inhibiting the release of mitochondrial DNA mediated by the NALP3 inflammasome. Nat. Immunol. 12, 222-230. doi: 10.1038/ni.1980

Natale, G., Pasquali, L., Paparelli, A., and Fornai, F. (2011). Parallel manifestations of neuropathologies in the enteric and central nervous systems. Neurogastroenterol. Motil. 23, 1056-1065. doi: 10.1111/j.1365-2982. 2011.01794.x

Obeso, J. A., Rodriguez-Oroz, M. C., Goetz, C. G., Marin, C., Kordower, J. H., Rodriguez, M., et al. (2010). Missing pieces in the Parkinson's disease puzzle. Nat. Med. 16, 653-661. doi: 10.1038/nm.2165

Okun, E., Griffioen, K. J., and Mattson, M. P. (2011). Toll-like receptor signaling in neural plasticity and disease. Trends Neurosci. 34, 269-281. doi: 10.1016/j.tins. 2011.02.005

Olanow, C. W. (2007). The pathogenesis of cell death in Parkinson's disease-2007. Mov. Disord. 22(Suppl. 17), S335-S342. doi: 10.1002/mds.21675
Pacheu-Grau, D., Gómez-Durán, A., Iglesias, E., López-Gallardo, E., Montoya, J., and Ruiz-Pesini, E. (2013). Mitochondrial antibiograms in personalized medicine. Hum. Mol. Genet. 22, 1132-1139. doi: 10.1093/hmg/dds517

Pallen, M. J. (2011). Time to recognise that mitochondria are bacteria? Trends Microbiol. 19, 58-64. doi: 10.1016/j.tim.2010.11.001

Pfeiffer, R. F. (2003). Gastrointestinal dysfunction in Parkinson's disease. Lancet Neurol. 2, 107-116. doi: 10.1016/S1474-4422(03)00307-7

Qian, Y., and Guan, M. X. (2009). Interaction of aminoglycosides with human mitochondrial $12 \mathrm{~S}$ rRNA carrying the deafness-associated mutation. Antimicrob. Agents Chemother. 53, 4612-4618. doi: 10.1128/AAC.00 965-08

Rabiet, M. J., Huet, E., and Boulay, F. (2007). The N-formyl peptide receptors and the anaphylatoxin C5a receptors: an overview. Biochimie 89, 1089-1106. doi: 10.1016/j.biochi.2007.02.015

Reichmann, H., Schneider, C., and Lohle, M. (2009). Non-motor features of Parkinson's disease: depression and dementia. Parkinsonism Relat. Disord. 15(Suppl. 3), S87-S92. doi: 10.1016/S1353-8020(09)70789-8

Sampson, T. R., Debelius, J. W., Thron, T., Janssen, S., Shastri, G. G., Ilhan, Z. E., et al. (2016). Gut microbiota regulate motor deficits and neuroinflammation in a model of Parkinson's disease. Cell 167, 1469.e12-1480.e12. doi: 10.1016/j.cell. 2016.11.018

Sana, T. G., Lugo, K. A., and Monack, D. M. (2017). T6SS: the bacterial "fight club" in the host gut. PLoS Pathog. 13:e1006325. doi: 10.1371/journal.ppat.1006325

Santos, D., Esteves, A. R., Silva, D. F., Januário, C., and Cardoso, S. M. (2015). The impact of mitochondrial fusion and fission modulation in sporadic Parkinson's disease. Mol. Neurobiol. 52, 573-586. doi: 10.1007/s12035-014-8893-4

Schapira, A. H. (2008). Mitochondria in the aetiology and pathogenesis of Parkinson's disease. Lancet Neurol. 7, 97-109. doi: 10.1016/S1474-4422(07) 70327-7

Scheperjans, F. (2018). The prodromal microbiome. Mov. Disord. 33, 5-7. doi: $10.1002 / \mathrm{mds} .27197$

Schroder, K., and Tschopp, J. (2010). The inflammasomes. Cell 140, 821-832. doi: 10.1016/j.cell.2010.01.040

Shen, J., Du, T., Wang, X., Duan, C., Gao, G., Zhang, J., et al. (2014). $\alpha$-Synuclein amino terminus regulates mitochondrial membrane permeability. Brain Res. 1591, 14-26. doi: 10.1016/j.brainres.2014.09.046

Shimada, K., Crother, T. R., Karlin, J., Dagvadorj, J., Chiba, N., Chen, S., et al. (2012). Oxidized mitochondrial DNA activates the NLRP3 inflammasome during apoptosis. Immunity 36, 401-414. doi: 10.1016/j.immuni.2012.01.009

Shintani, Y., Drexler, H. C., Kioka, H., Terracciano, C. M., Coppen, S. R., Imamura, H., et al. (2014). Toll-like receptor 9 protects non-immune cells from stress by modulating mitochondrial ATP synthesis through the inhibition of SERCA2. EMBO Rep. 15, 438-445. doi: 10.1002/embr.201337945

Stolzenberg, E., Berry, D., Yang, Lee, E. Y., Kroemer, A., Kaufman, S., et al. (2017). A role for neuronal alpha-synuclein in gastrointestinal immunity. J. Innate Immun. 9, 456-463. doi: 10.1159/000477990

Suzuki, M., Danilchanka, O., and Mekalanos, J. J. (2014). Vibrio cholerae T3SS effector VopE modulates mitochondrial dynamics and innate immune signaling by targeting Miro GTPases. Cell Host Microbe 16, 581-591. doi: 10.1016/j.chom. 2014.09.015

Svensson, E., Horváth-Puhó, E., Thomsen, R. W., Djurhuus, J. C., Pedersen, L., Borghammer, P., et al. (2015). Vagotomy and subsequent risk of Parkinson's disease. Ann. Neurol. 78, 522-529. doi: 10.1002/ana.24448

Taanman, J. W. (1999). The mitochondrial genome: structure, transcription, translation and replication. Biochim. Biophys. Acta 1410, 103-123. doi: 10.1016/ S0005-2728(98)00161-3

Takeuchi, O., and Akira, S. (2010). Pattern recognition receptors and inflammation. Cell 140, 805-820. doi: 10.1016/j.cell.2010.01.022

Tanner, C. M., Kamel, F., Ross, G. W., Hoppin, J. A., Goldman, S. M., Korell, M., et al. (2011). Rotenone, paraquat, and Parkinson's disease. Environ. Health Perspect. 119, 866-872. doi: 10.1289/ehp.1002839

Ward, A. E., and Rosenthal, B. M. (2014). Evolutionary responses of innate immunity to adaptive immunity. Infect. Genet. Evol. 21, 492-496. doi: 10.1016/ j.meegid.2013.12.021

Wilkins, H. M., Carl, S. M., Weber, S. G., Ramanujan, S. A., Festoff, B. W., Linseman, D. A., et al. (2015). Mitochondrial lysates induce inflammation and Alzheimer's disease-relevant changes in microglial and neuronal cells. J. Alzheimers Dis. 45, 305-318. doi: 10.3233/JAD- 142334 
Wilkins, H. M., Koppel, S. J., Weidling, I. W., Roy, N., Ryan, L. N., Stanford, J. A., et al. (2016). Extracellular mitochondria and mitochondrial components act as damage-associated molecular pattern molecules in the mouse brain. J. Neuroimmune Pharmacol. 11, 622-628. doi: 10.1007/s11481-0169704-7

Wilkins, H. M., Weidling, I. W., Ji, Y., and Swerdlow, R. H. (2017). Mitochondriaderived damage-associated molecular patterns in neurodegeneration. Front. Immunol. 8:508. doi: 10.3389/fimmu.2017.00508

Xiong, N., Long, X., Xiong, J., Jia, M., Chen, C., Huang, J., et al. (2012). Mitochondrial complex I inhibitor rotenone-induced toxicity and its potential mechanisms in Parkinson's disease models. Crit. Rev. Toxicol. 42, 613-632. doi: 10.3109/10408444.2012.680431

Zhang, Q., Raoof, M., Chen, Y., Sumi, Y., Sursal, T., Junger, W., et al. (2010). Circulating mitochondrial DAMPs cause inflammatory responses to injury. Nature 464, 104-107. doi: 10.1038/nature08780
Zhou, R., Yazdi, A. S., Menu, P., and Tschopp, J. (2011). A role for mitochondria in NLRP3 inflammasome activation. Nature 469, 221-225. doi: 10.1038/ nature 09663

Conflict of Interest Statement: The authors declare that the research was conducted in the absence of any commercial or financial relationships that could be construed as a potential conflict of interest.

Copyright (c) 2018 Cardoso and Empadinhas. This is an open-access article distributed under the terms of the Creative Commons Attribution License (CC BY). The use, distribution or reproduction in other forums is permitted, provided the original author(s) and the copyright owner are credited and that the original publication in this journal is cited, in accordance with accepted academic practice. No use, distribution or reproduction is permitted which does not comply with these terms. 